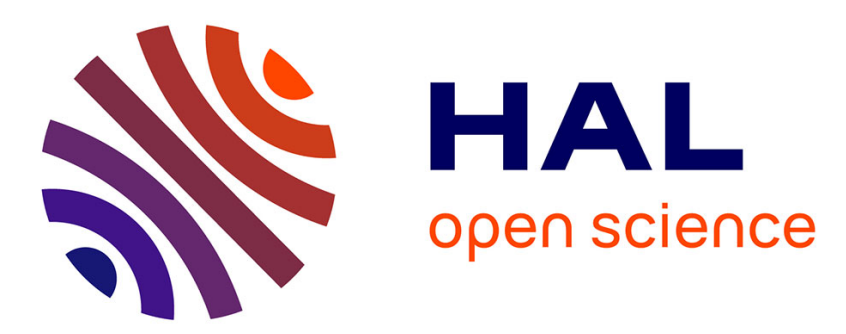

\title{
XAFS Study of the Antiferroelectric Phase Transition in $\mathrm{PbZrO3}$
}

\author{
N. Sicron, Y. Yacoby, E. Stern, F. Dogan
}

\section{To cite this version:}

N. Sicron, Y. Yacoby, E. Stern, F. Dogan. XAFS Study of the Antiferroelectric Phase Transition in PbZrO3. Journal de Physique IV Proceedings, 1997, 7 (C2), pp.C3-1047-C3-1049. 10.1051/jp4:19972134 . jpa-00255195

\section{HAL Id: jpa-00255195 https://hal.science/jpa-00255195}

Submitted on 1 Jan 1997

HAL is a multi-disciplinary open access archive for the deposit and dissemination of scientific research documents, whether they are published or not. The documents may come from teaching and research institutions in France or abroad, or from public or private research centers.
L'archive ouverte pluridisciplinaire HAL, est destinée au dépôt et à la diffusion de documents scientifiques de niveau recherche, publiés ou non, émanant des établissements d'enseignement et de recherche français ou étrangers, des laboratoires publics ou privés. 


\title{
XAFS Study of the Antiferroelectric Phase Transition in $\mathbf{P b Z r O}_{3}$
}

\author{
N. Sicron, Y. Yacoby, E.A. Stern* and F. Dogan** \\ Racah Institut of Physics, Hebrew University of Jerusalem, Jerusalem 91904, Israel \\ * Physics Department FM-15, University of Washington, Seattle, WA 98195, U.S.A. \\ ** Department of Materials Science and Engineering, University of Washington, Seattle, \\ WA 98195, U.S.A.
}

\begin{abstract}
The temperature dependence of the local structure of $\mathrm{PbZrO}_{3}(\mathrm{PZ})$ was investigated using XAFS analysis. This perovskite undergoes an antiferroelectric (AFE) phase transition at about $500 \mathrm{~K}$. XAFS of $\mathrm{Pb} \mathrm{L}_{\mathrm{III}}$-edge and Zr K-edge in PZ were measured between $173 \mathrm{~K}$ and $824 \mathrm{~K}$. The local structure was determined by fitting a parameterized theoretical XAFS to the experimental data. The distortion parameters obtained at room temperature are consistent with previous diffraction results. The $\mathrm{Pb}[1,1,0]$ displacement remains almost constant at all temperatures. The $\mathrm{ZrO}_{6}$ octahedron remains distorted at all temperatures but the exact distortion above $T_{c}$ cannot be determined. The oxygen AFE distortion and the $[1,1,0]$ octahedral rotation decrease as temperature increases. At $824 \mathrm{~K}$ the oxygen distortions are at least $50 \%$ of their $298 \mathrm{~K}$ values. The presence of structural distortions in the paraelectric phase of $\mathrm{PZ}$ indicates that like in many other perovskites these distortions are not a result of soft lattice vibrational modes and that the AFE phase transition involves the ordering of local distortions.
\end{abstract}

\section{INTRODUCTION}

The perovskite Lead Zirconate (PZ) undergoes an antiferroelectric (AFE) phase transition at about $500 \mathrm{~K}$ [1]. The distortion from the cubic paraelectric (PE) structure can be decomposed into several atomic displacements, associated with different lattice vibrational modes [2]. In PZ there are five such displacements: anti parallel $[1,1,0]$ displacements of $\mathrm{Pb}$ and $\mathrm{Zr}$ atoms (all the vectors are in the pseudo cubic coordinate system), anti parallel $[-1,-1,0]$ displacement of $\mathrm{O}_{1}$ atoms (oxygens in the $\mathrm{Pb}$ plain), a more complicated displacement of $\mathrm{O}_{2}$ atoms and rotation of the oxygen octahedra around the $[1,1,0]$ axis. A rhombohedral ferroelectric (FE) intermediate phase was also reported for PZ. The temperature range and the stability of the intermediate phase are very sensitive to the presence of impurities in the crystal and to the preparation method [1].

The AFE transition in PZ was described as a displacive transition [2]. In the displacive model the transition is from an ordered PE phase to an ordered phase with lower symmetry. The structural distortion is caused by the softening of vibrational lattice mode (or modes). In the recent years experimental evidence, especially from XAFS have shown that local distortions exist in the PE phase of many perovskites which were previously considered as displacive. Among these are the ferroelectrics $\mathrm{KNbO}_{3}$ [3], $\mathrm{KNb}_{x} \mathrm{Ta}_{1-\mathrm{x}} \mathrm{O}_{3}$, [4] and $\mathrm{PbTiO}_{3}$ [5] and the antiferrodistortive $\mathrm{Na}_{\mathrm{x}} \mathrm{K}_{1-x} \mathrm{TaO}_{3}$ [6]. So far no AFE transition in perovskites has been studied by XAFS. In this paper we present a study of the temperature dependence of the local distortions in PZ. We show that like in other perovskites studied previously there are local distortions in the PE phase of PZ.

\section{EXPERIMENTAL CONDITIONS AND DATA ANALYSIS}

PZ sample was prepared by a sol-gel process. XRD measurement verified its orthorhombic structure. Dielectric measurement showed a single phase transition at $499 \mathrm{~K}$ on heating and at $498 \mathrm{~K}$ on cooling. $\mathrm{Pb} \mathrm{L}_{\mathrm{III}}$-edge and $\mathrm{Zr} \mathrm{K}$-edge XAFS were measured at seven different temperatures between 173 and $824 \mathrm{~K}$. All data was collected at beamline X-11A at the NSLS. Full experimental details can be found elsewhere [7]. The background was removed in a procedure described in ref. 5 . The

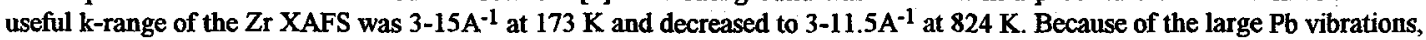
the $\mathrm{Pb}$ XAFS quality was not as good as the $\mathrm{Zr}$ spectra. For $\mathrm{Pb}$ XAFS the useful k-range was $3-12 \mathrm{~A}^{-1}$ at $173 \mathrm{~K}$ and decreased to $3-10 \mathrm{~A}^{-1}$ at $824 \mathrm{~K}$.

The analysis is based of fitting the Fourier transforms (FT) of theoretical calculations to the FT of experimental data in a specified range in $\mathrm{r}$-space [4]. The theoretical XAFS was expressed in the form:

$$
\chi(k)=\operatorname{Im} \sum_{\eta} n_{\eta} F_{\eta}(k) \exp \left\{i\left[\frac{B_{\eta}}{k}+k L_{\eta}+\theta_{\eta}(k)-\frac{4}{3} k^{3} C_{3 \eta}\right]-2 k^{2} \sigma_{\eta}{ }^{2}-\frac{L_{\eta}}{\lambda_{\eta}}\right\}
$$

Here the summation is over all different scattering paths (indexed by $\eta$ ), $n$ is number of equivalent paths, $k$ is the photoelectron's wave number, $L$ is the scattering path length, $F(k)$ and $\theta(k)$ are the scattering amplitude and phase which were calculated by FEFF5 code [8]. $B / k$ and $L / \lambda$ are phase shifts and mean free path correction terms. $\sigma^{2}$ and $C_{3}$ are the second and third cumulants of the radial distribution function of the atoms in a given shell (or sub shell) relative to the probe 
atom [5]. Errors in the fit parameters were estimated from the covariance matrix of the Levenberg-Marquardt non linear fit [9]. The threshold energy $E_{0}$ was set at the mid point of the absorption step. For each probe atom the $B^{\prime}$ s and $\lambda^{\prime}$ swere determined by fitting the data of the two lowest temperatures (173 and 298k) and then were set constant for all the data.

The $\mathrm{Zr}$ spectra were fitted between 1.0 and $4.2 \mathrm{~A}$, using the four nearest coordination shells $(\mathrm{O}, \mathrm{Pb}, \mathrm{Zr}$ and $\mathrm{O})$. The calculation included the double and triple collinear scattering of the $\mathrm{Zr}-\mathrm{O}-\mathrm{Zr}$ path. Three other double scattering paths were also included. The second moment of the rotation and the oxygen AFE distortion (or their rms values) are obtained from the affect of these distortions on the collinear scattering of the third $\mathrm{Zr}$ shell in the $\mathrm{Zr}$ XAFS [6]. Because of thermal vibrations the rms distortions are expected to be larger than the first moment of the distortion. (The first moments of distortions are obtained from their affect on the bond lengths of the first and fourth shells). Since the two oxygen distortions affect the same shells in a similar manner the oxygen distortion parameters in our parameter fit depend on each other. This conld lead to errors in these parameters. Five $\sigma^{2}$ were used: one for each shell, except for the second $\mathrm{Pb}$ shell in which two $\sigma^{2}$ were used, one for the two near $\mathrm{Pb}$ atoms and one for the other six. The distortions were described with six parameters: AFE distortion of $\mathrm{Pb}$ and $\mathrm{Zr}$, oxygen $\mathrm{AFE}$ distortion (it was assumed that the ratio between the $\mathrm{O}_{1}$ and $\mathrm{O}_{2} \mathrm{AFE}$ distortions does not change with temperature and one parameter controlled both of them), octahedral rotation, and the rms rotation and AFE distortions. After fitting all the data with 11 parameters the $\sigma^{2}$ obtained were fitted to Einstein models. The refinement of the six structural parameters was done by setting the $\sigma^{2}$ to the values obtained in the Einstein model fit.

The $\mathrm{Pb}$ spectra were fitted between 1.0 and 3.7A (1.0-4.75A at low temperatures), using the four nearest shells $(\mathrm{O}, \mathrm{Zr}$, $\mathrm{Pb}$ and $\mathrm{O}$ ) and one double scattering path, $\mathrm{Pb}-\mathrm{O}-\mathrm{Zr}$. The third and fourth shell were not sensitive the distortions and were included mainly to avoid errors in the second $\mathrm{Zr}$ shell parameters. In the AFE phase of $\mathrm{PZ}$ there are two non equivalent $\mathrm{Pb}$ probe atoms and the theoretical calculations included the sum of the XAFS of the two Pb probes. Six $\sigma^{2}$ were used: two for the first oxygen shell, two for the second $\mathrm{Zr}$ shell (as in the $\mathrm{Zr}$ XAFS) and one for the third and fourth shells. A $C_{3}$ parameter was used for the short $\mathrm{Pb}-\mathrm{O}$ bonds. The $\mathrm{Pb}$ displacement cannot be determined from the first oxygen shell of the $\mathrm{Pb}$ XAFS [7]. The $\mathrm{Pb}$ XAFS provides only the relative $\mathrm{Pb}-\mathrm{Zr}[1,1,0]$ displacement. Following the results of $\mathrm{Zr}$ XAFS analysis the $\mathrm{Zr}$ displacement was set to $0.04 \mathrm{~A}$ at all temperatures and only three distortion parameters were variable. These were the $\mathrm{Pb}$ displacement, the oxygen AFE distortion and octahedral rotation. The refinement of the structural parameters was done by setting the $\sigma^{2}$ of the far $\mathrm{Zr}$ and the third and fourth shells to Einstein model values. The other $\sigma^{2}$ do not fit the Einstein model and were left variable. Typical fits are presented in fig. 1 .
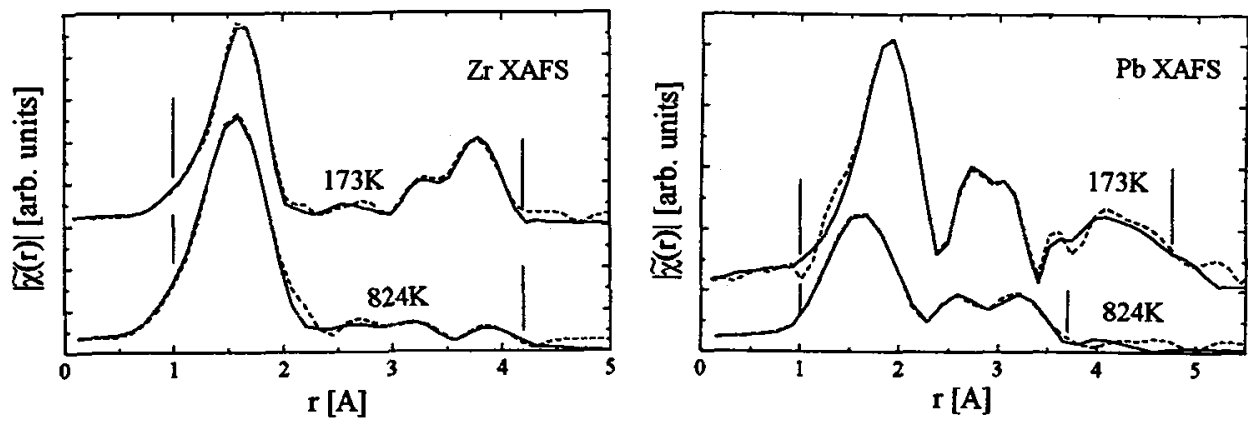

Figure 1: Examples of the absolute value of $k^{2}$ weighted FT of the $\mathrm{Zr}$ and $\mathrm{Pb}$ XAFS. Dashed lines - experimental data, solid lines - best fit. The vertical lines indicate the fitting range. The FT were taken from different k-ranges for different temperatures. Therefore their magnitudes are not comparable.

\section{RESULTS AND DISCUSSION}

The $\mathrm{Zr}$ and $\mathrm{Pb}[1,1,0]$ displacements are presented in fig. 2. The displacements at room temperature are consistent with previous results [2]. The slight increase in the $\mathrm{Zr}$ displacement is not significant. The magnitude of the second $\mathrm{Pb}$ shell in the $\mathrm{Zr}$ XAFS is relatively small. This causes large uncertainties in all the $\mathrm{Pb}$ parameters obtained from the $\mathrm{Zr} \mathrm{XAFS}$. The $\mathrm{Pb}$ displacements obtained form the two probes are consistent with each other. The difference between the local $\mathrm{Pb}$ displacement and the macroscopic average displacement obtained from XRD near $\dot{T}_{c}[1]$, indicates that even in the AFE phase there is partial structural disorder. The relative $\mathrm{Pb}-\mathrm{Zr}$ displacement decreases only slightly at high temperatures. A similar behavior was observed in $\mathrm{PbTiO}_{3}[5]$ where the relative $\mathrm{Pb}-\mathrm{Ti}$ [1,0,0] displacement was constant at all temperatures.

The temperature dependence of the $[1,1,0]$ octahedral rotation angle is presented in fig. 3 . Below $T_{c}$ the results of both probes are consistent with each other and with XRD results [1]. The rms rotation angle is slightly larger because of thermal vibrations. Above $\mathrm{T}_{\mathrm{c}}$ the angles obtained from both probes are different from each other. In the $\mathrm{Pb}$ XAFS the rotation angle is defermined mainly form the short $\mathrm{Pb}-\mathrm{O}_{2}$ bond length. This bond is also affected by the oxygen AFE distortion and an error in the AFE distortion parameter would lead to an error in the rotation parameter. The correlation between the oxygen distortion parameters in the Zr XAFS leads to large errors in the rms and the average rotation angles. The discrepancies between the rotation parameters might suggest that the local oxygen structure above $T_{c}$ cannot be described by the low temperature 
distortions. Nevertheless the perpendicular oxygen displacements decrease graduaily as temperature increases but remain finite above $T_{c}$ in contrast to the average distortions which vanish in the PE phase. The AFE oxygen distortion decreases by $20 \%$ from $173 \mathrm{~K}$ to $824 \mathrm{~K}$. This distortion will not be discussed here.

We tried to fit several other structural models to the data of the PE phase in order to test the significance of our results. A cubic structure with no distortions cannot fit the $\mathrm{Pb}$ XAFS at all. In this case the calculated FT of the oxygen first shell is in anti phase with the experimental FT. A structure with variable $\mathrm{Pb}$ and $\mathrm{Zr}$ displacements but no oxygen distortions can fit the $\mathrm{Pb}$ XAFS but the fit is 10 times worse than the fit of the low temperature model. Two models were tested for the first oxygen shell in the Zr XAFS: a cubic structure with no distortions and a model with a $\mathrm{Zr}[1,1,1]$ displacement (in both models a $C_{3}$ parameter was included). The misfit of these models is about $7 \%$ for the cubic model and $4 \%$ for the [1,1,1] model. The misfit for the $[1,1,0]$ model is $3 \%$. Thus, the cubic model can be excluded for the $\mathrm{ZrO}_{6}$ octahedron but we cannot distinguish between the $[1,1,0]$ and $[1,1,1]$ models. A model with $\mathrm{Pb}$ and $\mathrm{Zr}[1,1,1]$ displacements and $[1,1,1]$ octahedral rotations can fit the data from both probes, above $\mathrm{T}_{\mathrm{c}}$. However, the $\mathrm{Pb}-\mathrm{Zr}$ relative displacement obtained from the two probes are significantly different form each other.

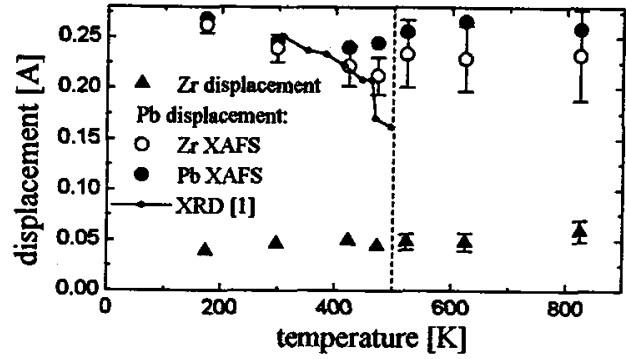

Figure 2: $\mathrm{Pb}$ and $\mathrm{Zr}[1,1,0]$ displacements as a function of the temperature. The errors in the $\mathrm{Zr}$ displacement below $\mathrm{T}_{\mathrm{c}}$ and in the $\mathrm{Pb}$ displacement obtained from the $\mathrm{Pb}$ XAFS are of the order of the points in the figure. The vertical dashed line indicates $T_{c}$.

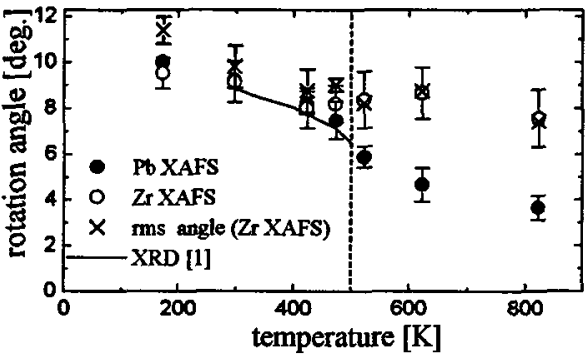

Figure 3: The rotation angle of the oxygen octahedron as a function of temperature. The rotation angle in ref. 1 was deduced from the tetragonal distortion of the pseudo cubic unit cell. The vertical dashed line indicates $\mathrm{T}_{c}$.

The results and conclusions of this study can be summarized as follows:

1) At room temperature the distortion parameters obtained by XAFS are consistent with the results of $x$-ray [1] and nentron [2] diffraction.

2) $\mathrm{The} \mathrm{Pb}[1,1,0]$ displacement remains almost constant up to $824 \mathrm{~K}$. The $\mathrm{ZrO}_{6}$ octahedron remains distorted above $\mathrm{T}_{c}$ but its exact structure can not be determined from XAFS.

3) The perpendicular oxygen displacements (AFE distortion and rotations) decrease as temperature increases but do not vanish at all temperatures.

4) Since all local distortions in $P Z$ remain finite more than 300 degrees above $T_{c}$ the local distortions are not caused by soft modes. The transition to the AFE phase involves ordering of the local distortions.

\section{Acknowledgments}

This work was supported in part by DOE Grant No. DE-FG06-90ER45425 and BSF Grant No. 92-00348. Beamline X11-A is supported by DOE Grant No. DE-FG05-89ER45384. We wish to thank Dr. Yu. Feldman for the dielectric measurements.

\section{References}

[1] Whatmore R.W. and Glazer A.M., J. Phys. C: Solid State Phys. 12 (1979) 1505-1518.

[2] Fujishita H., and Hoshino S., J. Phys. Soc. Jap. 53 (1984) 226-234.

[3] Kim K.H., Elam W.T. and Skelton E.F., Matter. Res. Soc. Symp. Proc. 172 (1990) 291-294.

[4] Hanske-Petitpierre O., Yacoby Y., Mustre-deleon J., Stern E.A. and Rehr J.J., Phys. Rev. B 44 (1991) 6700-6707.

[5] Sicron N., Ravel B., Yacoby Y., Stern E. A., Dogan F., and Rehr J.J., Phys. Rev. B 50 (1994) 13168-13180.

[6] Rechav B., Yacoby Y., Stern E. A., Rehr J.J. and Newville M., Phys. Rev. Lett. 72 (1994) 1352-1355.

[7] Sicron N., Yacoby Y., Stern E. A., unpublished.

[8] Rehr J.J., Albers R.C., and Zabinsky S.I., Phys. Rev. Lett. 69 (1992) 3397-3400.

[9] Press W.H., Flannery B.P., Teukolsky S.A., and Vetterling W.T., Numerical Recipes, (Cambridge Univ. Press, Cambridge, 1986) p. 536. 